\title{
Obtenção das constantes elásticas efetivas sob condição dinâmica de um laminado híbrido para uso aeronáutico
}

\author{
Obtaining the effective elastic constants under \\ dynamic condition of hybrid laminate for \\ aeronautical use
}

Carlos Alberto Rios Brito Júnior ${ }^{1}$, Luis Claudio Pardini ${ }^{2}$, Antônio Carlos Ancelotti Júnior ${ }^{3}$

\author{
${ }^{1}$ Coordenação da Engenharia Aeroespacial/ CCET/ UFMA - Av. dos Portugueses, 1966, Bacanga, CEP 65080-805, São \\ Luís, MA, Brasil. \\ ${ }^{2}$ Divisão de Materiais - AMR/ IAE/ DCTA, São José dos Campos, SP, Brasil. \\ ${ }^{3}$ Instituto de Engenharia Mecânica, Universidade Federal de Itajubá, Itajubá, MG, Brasil. \\ e-mail: riosbritojunior@yahoo.com.br, pardini@iae.cta.br, antonio.ancelotti@gmail.com
}

\section{RESUMO}

O presente trabalho aborda a determinação das constantes elásticas efetivas de materiais compósitos estruturais de uso aeronáutico por meio de ensaio de vibração. O experimento foi conduzido em um aparato apropriado com interferômetro a laser. Inicialmente, foi realizado um ensaio de vibração do tipo livre para obtenção das frequências naturais intrínsecas à placa de compósito híbrido de alumínio aeronáutico com tecido prepreg de fibra de carbono/epóxi (CARALL). Do mesmo modo, verificou-se por meio deste método, o alumínio 2024 T3 que é uma liga metálica que pode ser aplicada em aeronaves. Tanto o alumínio quanto os compósitos foram modelados em aplicativo computacional (ANSYS) onde por meio do método de elementos finitos obtiveram-se frequências relacionadas aos seis primeiros modos de vibração. O alumínio aeronáutico apresentou uma maior precisão entre os materiais analisados em relação as suas frequências naturais. Em relação as constantes elásticas, a liga de alumínio 2024 T3 apresentou um módulo $\mathrm{E}_{1}$ de 71,3 GPa e um coeficiente de Poisson de 0,31 . As constantes elásticas do CARALL foram determinadas como solução de um problema de otimização computacional descrita em linguagem própria contida em uma sub-rotina do ANSYS. Observou-se que o módulo de elasticidade principal e o coeficiente de Poisson são próximos dos valores encontrados numericamente pela regra da mistura (70 GPa e 0.3 , respectivamente).

Palavras-chave: Compósito híbrido, fibras de carbono, ensaio de vibração, propriedades mecânicas

\begin{abstract}
This paper deals with the determination of the effective elastic constants of structural composite materials of aeronautical use by means of vibration test. The experiment was conducted in an appropriate apparatus with a laser interferometer. Initially, a free-vibration mode vibration test was performed to obtain the natural frequencies intrinsic to the hybrid composite plate (aeronautical aluminum lamina's alternating to carbon fiber/epoxy fabric lamina's) known as CARALL. Likewise, aluminum $2024 \mathrm{~T} 3$ which is a metallic alloy that can be applied in aircraft was verified by means of this method. Both the aeronautical aluminum and the hybrid composite were modeled in a computer application (ANSYS) where, through the finite element method, frequencies related to the first six modes of vibration were obtained. The aeronautical aluminum presented a greater precision between the materials analyzed in relation to their natural frequencies. Regarding the elastic constants, the $2024 \mathrm{~T} 3$ aluminum alloy presented an $\mathrm{E}_{1}$ modulus of $71.3 \mathrm{GPa}$ and a Poisson's ratio of 0.31 . The CARALL elastic constants were determined as the solution of a computational optimization problem described in its own language contained in an ANSYS subroutine. It was observed that the main modulus of elasticity and Poisson's coefficient are close to the values found numerically by the mixing rule ( $70 \mathrm{GPa}$ and 0.3 , respectively).
\end{abstract}

Keywords: Hybrid composite, carbon fiber, vibration test, mechanical properties 


\section{INTRODUÇÃO}

O termo "híbrido" para compósito surgiu no emprego de laminados que utilizam tecidos fabricados pela combinação de diferentes tipos de fibras. Ou seja, lâminas (comumente tecido impregnado com resina) de fibras de carbono e fibras de vidro, por exemplo, podem ser usadas para configurar um compósito híbrido, porém, não há somente este tipo de configuração. Os compósitos metal/fibra podem ser considerados outro tipo de compósito híbrido e, especificamente, podem ser denominados de laminados metal-fibra (FML) [1]. Inicialmente, os FML's foram desenvolvidos para aplicação aeronáutica nos estudos de Jaap Schijve e Boud Vogelesang, pesquisadores da Universidade de Delft (Holanda) em 1978, onde simularam voos com uma aeronave construída com laminados reforçados com fibras de aramida e carbono [2]. Os FML's concebem propriedades mecânicas peculiares de cada constituinte em um único sistema. Um material com alta tolerância a danos, resistência a corrosão, alta rigidez específica e propagação de trincas por fadiga, o que o torna um atrativo a indústria de aeronaves [3-6].

Diversos experimentos e métodos numéricos foram aplicados para se determinar as propriedades mecânicas semi-estáticas para compósitos tipo FML [7-10]. Em particular, no caso do CARALL (acrônimo para CArbon Reinforced Aluminum Laminates), quando submetido aos ensaios mecânicos de tensão, por exemplo, podem fornecer constantes elásticas com valores próximos aos obtidos teoricamente, ou seja, $\mathrm{E}_{\mathrm{x}}=\mathrm{E}_{\mathrm{y}}=72 \mathrm{GPa}, v_{\mathrm{xy}}=0,20$ e $\mathrm{G}_{\mathrm{xy}}=18 \mathrm{GPa}[8,9]$. Entretanto, os resultados para propriedades mecânicas dos FML's podem ser influenciados principalmente por características como os constituintes dos compósitos, tipo de metal e tipo de fibra, sistema adesivo, tratamento de superfícies metálicas, fração volumétrica fibra/metal, entre outros fatores [7]. Sasso e colaboradores [10] verificaram que ao adotar o formato de corpo de prova conforme a norma ASTM D3039, o módulo de elasticidade na direção das fibras sob ensaio de tração $\left(\mathrm{E}_{1} \approx 60,1\right.$ $\mathrm{GPa}$ ) se diferencia do módulo transversal que sofre redução sob tensões maiores que $170 \mathrm{MPa}$ ( $\mathrm{E}_{2}$ para até $\approx 25,6 \mathrm{GPa}$ ).

Atualmente, se emprega o FML em painéis para fuselagem de aeronaves e em flapes. Entretanto, somente se justifica o uso do FML em substituição ao alumínio aeronáutico, por exemplo, se este compósito apresentar vantagens significativas em relação ao peso estrutural e comportamento mecânico quando submetido à condição dinâmica. Assim, a compreensão da natureza do amortecimento promove adaptação dos projetos mediante as solicitações operacionais [7].

O presente trabalho empregou uma metodologia experimental numérica na tentativa de simular as condições dinâmicas de painéis empregados na fuselagem de uma aeronave. Por meio da técnica de vibração em uma placa livre-livre foi possível encontrar as frequências modais que trazem as informações sobre o amortecimento do FML estudado. Essas frequências experimentais foram usadas para determinar indiretamente as constantes elásticas de um FML para possível uso aeronáutico. Portanto, a motivação deste trabalho foi o levantamento das propriedades elásticas em resposta dinâmica para formação de um banco de dados permissíveis de projeto.

\section{MATERIAIS E MÉTODOS}

\subsection{Materiais}

Os materiais empregados neste trabalho foram providenciados pela Divisão de Materiais AMR do Instituto de Aeronáutica e Espaço IAE/DCTA. Como se trata de um ensaio não destrutivo não foi necessário usar numerosos corpos de prova, somente uma placa plana retangular para cada material analisado foi o suficiente.

A liga metálica especificada como AA2024-T3 apresenta composição de 91,9\% de alumínio com 4,3\% de cobre solubilizado, trabalhado a frio e envelhecido naturalmente até uma condição substancialmente estável. Apresenta em média, propriedades mecânicas como módulo de elasticidade de $72 \mathrm{GPa}$, módulo de cisalhamento de $28 \mathrm{GPa}$, coeficiente de Poisson de 0,33 e massa específica de $2700 \mathrm{~kg} / \mathrm{m}^{3}$ [8]. Para o ensaio de vibração livre, empregou-se uma placa desta liga com dimensões de (300x200) $\mathrm{mm}$ e espessura de $6,5 \mathrm{~mm}$.

A principal placa teste para este ensaio foi o compósito híbrido tipo FML denominado de CARALL. Essa placa foi constituída por três lâminas de alumínio AA2024-T3 (com 0,4 mm de espessura) intercaladas por prepreg de fibra de carbono/resina epóxi. A manufatura deste compósito pode ser resumida em: (i) Tratamento químico superficial das lâminas de alumínio por anodização crômica para promover maior rugosidade da superfície metálica (consequentemente maior adesão) e garantir a proteção corrosiva necessária na presença de materiais dissimilares (fibras de carbono e resina epóxi); (ii) Empilhamento das lâminas sob bolsa de vácuo formando o compósito desejado, e; (iii) Ciclo de cura em autoclave com temperatura de $120^{\circ} \mathrm{C}$ e pressão de $0,7 \mathrm{MPa}$. A placa de CARALL após o desmolde foi seccionada para as dimensões de (300x200) mm. A espessura final do compósito determinada por micrografia de sua seção transversal ficou em 1,66 mm. Considerou-se, portanto, este compósito como uma placa fina. Outra consideração foi quanto à fração volumétrica do CARALL em estudo. Como a placa de compósito foi obtida em autoclave dentro de bolsa de vácuo, praticamente pode se 
desprezar a fração volumétrica de vazios $\mathrm{V}_{\mathrm{v}}$ que deve ser inferior a 1\% [9]. Assim, as frações em volume determinadas para fibras, resina e alumínio foram, respectivamente, nos valores de $V_{\text {fbra }}=15 \%, V_{\text {resina }}=13 \%$ (ambas informadas pelo fornecedor do prepreg, Hexcel Composites) e $V_{A l}=72 \%$. A massa específica do CARALL obtida com auxílio de uma balança analítica de precisão $0,001 \mathrm{~g}$ foi de $2510 \mathrm{~kg} / \mathrm{m}^{3}$.

\subsection{Metodologia experimental}

O método de ensaio por vibração livre-livre em placas fornece resultados a partir de uma função de resposta em frequência (FRF). Comumente, para descrição de um mecanismo com múltiplos graus de liberdade emprega-se um modelo modal com várias frequências naturais e suas correspondentes formas modais [7]. As FRF podem ser obtidas via experimental pela simples observação das curvas de magnitude fornecida em escala logarítmica.

No ensaio de vibração, a placa foi pendurada por fios de Nylon amarrados em molas fixadas em um suporte. A placa sofreu excitação por um vibrador eletrodinâmico (shaker) em um único ponto nodal (driving point). Cada ponto nodal forneceu valores relativos à sua magnitude e ao seu autovalor (frequência ressonante, em Hz) necessários para obter as FRF's para os primeiros modos de vibração. O equipamento necessário para realizar este ensaio encontra-se no Instituto de Aeronáutica e Espaço, IAE/DCTA, cidade de São José dos Campos - SP. Trata-se de dois sistemas independentes e conectados. O sistema de excitação responsável por transmitir a frequência de vibração à placa e o sistema de medição e processamento de dados que promove a geração e varredura da malha com auxílio de um sensor a laser (Figura 1).

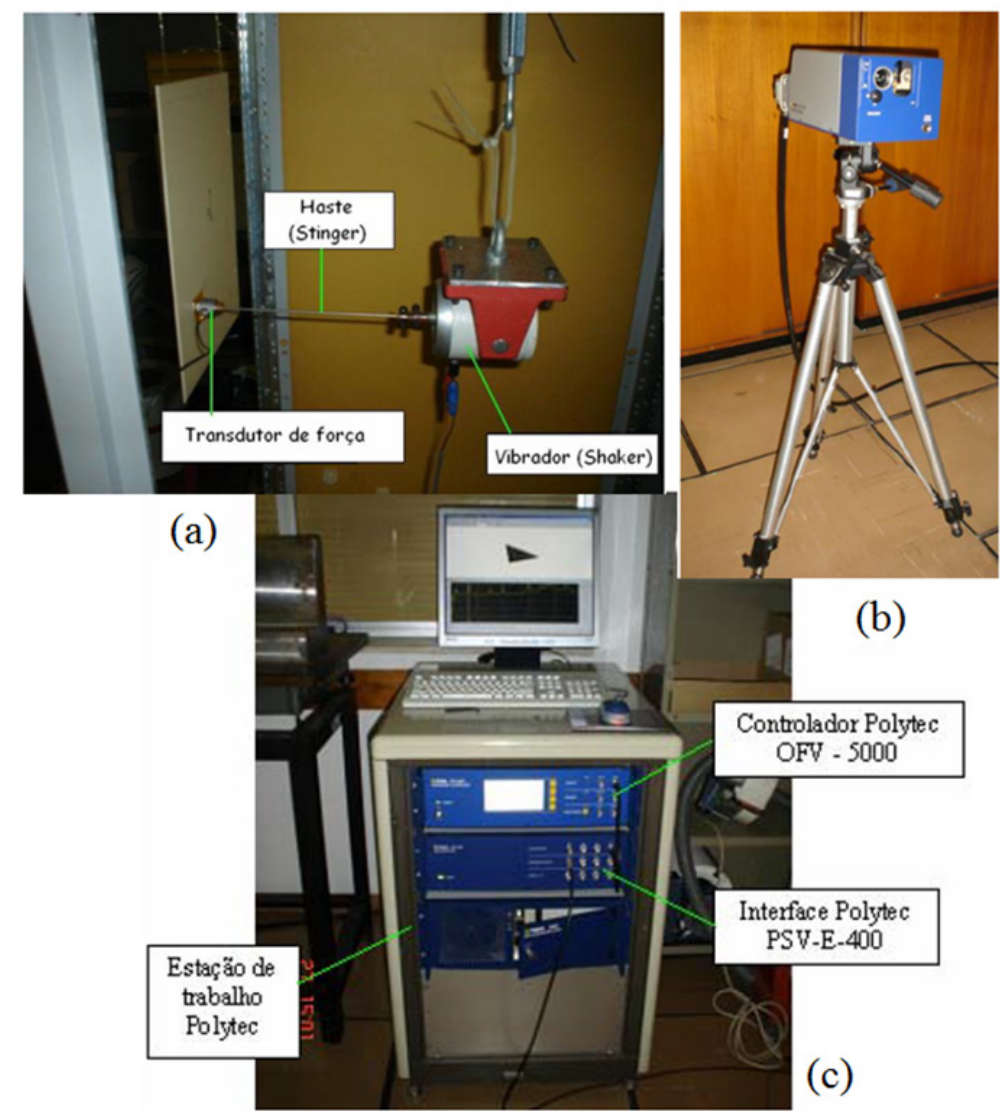

Figura 1: (a) Dispositivos do sistema de excitação. (b) Sensor a laser. (c) Estação de medição e processamento de dados. (adaptado de Brito Júnior [11])

As frequências experimentais foram obtidas a partir da média das frequências fornecidas pela FRF de cada nó. A malha gerada durante ensaio para a placa teste CARALL foram arbitradas em 25 pontos nodais, enquanto, para a placa de alumínio foi gerado 81 nós. Para cada nó, foram fornecidos os dados relativos à sua magnitude e ao seu autovalor (frequência ressonante). Então, foram processados 25 gráficos de resposta em função da frequência para o CARALL e 81 gráficos para o alumínio, todos na faixa de $0,25 \mathrm{~Hz}$ até $800,25 \mathrm{~Hz}$ com variação de $0,05 \mathrm{~Hz}$. Utilizou-se o aplicativo computacional Origin para gerar estes gráficos (conforme Figura 2). 


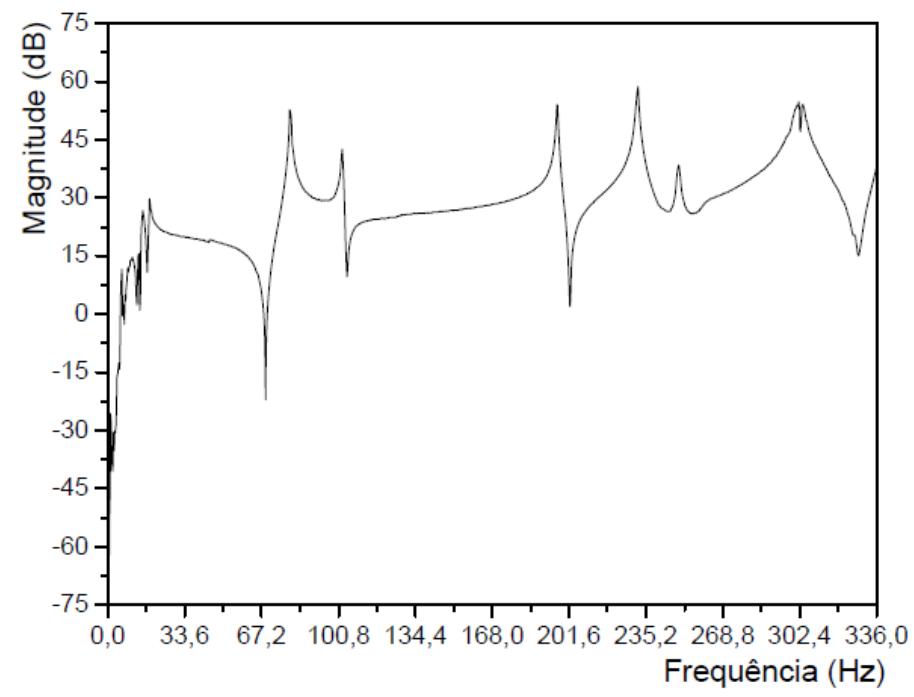

Figura 2: Gráfico FRF para os seis primeiros modos de vibração da placa de CARALL obtidas num ponto nodal [7].

\subsection{Metodologia numérica}

O método de elementos finitos MEF tem sido preferencialmente adotado para modelagem do comportamento dinâmico de placas de compósitos com mais de uma lâmina, por apresentar vantagens sobre outros modelos numéricos, como por exemplo, o método de Ritz [10]. Dentre as principais vantagens do MEF podem-se mencionar: (a) a simplificação do processo de modelagem via computacional; (b) baixo consumo de tempo devido a capacidade atual de processamento dos computadores, e; (c) facilidade em controlar as condições de contorno e as formas complexas das placas [12].

Foi empregado o programa computacional ANSYS para realizar a modelagem das placas de compósitos híbridos deste estudo. Este programa apresenta uma sub-rotina para otimização de projeto empregando como método numérico o MEF. Nesta sub-rotina foram inseridos os parâmetros modais e informações do material obtidas no ensaio experimental de vibração livre-livre. Com os limites e tolerâncias estabelecidos previamente para definir o espaço de projeto factível (que não corrompe nenhuma restrição) é possível aproximar as frequências numéricas calculadas no programa com as frequências experimentais por meio da minimização de uma função objetivo $\mathrm{F}_{\text {objt }}$. Lembrando que as frequências experimentais usadas na otimização de projeto do programa ANSYS foram obtidas a partir da média das frequências fornecidas pela FRF de cada nó ou ponto nodal. Consequentemente, as constantes elásticas, que tem seus valores iniciais arbitrados, são obtidas via essa aproximação entre as variáveis de estado que são dependentes destas constantes elásticas. Trata-se, portanto, de uma conversão de um problema de minimização restrito para irrestrito executado por uma função penalidade em todo processo de interação. Este método de minimização é denominado de método de aproximação por Subproblema. Resumidamente, o cálculo das constantes elásticas pode ser descrito a seguir [7, 13]:

- A geração da malha, o cálculo das frequências numéricas e a otimização do processo são formalizados em uma linguagem própria do ANSYS. Essa linguagem denominada de APDL (Ansys Parametric Design Language) faz parte do módulo "Design Optimization".

- O processo de projeto, seguindo as especificações deste programa, é caracterizado pelas variáveis de otimização (variáveis de projeto, variáveis de estado e função objetivo) que são parâmetros escalares na linguagem APDL e estão sujeitas a restrições (limites de tolerâncias) definido assim o espaço de projeto factível (ou aceitável) de onde as configurações do projeto não pode violar nenhuma das restrições;

- Neste caso, as variáveis de projeto correspondem as constantes elásticas que se deseja encontrar, enquanto, as variáveis de estado, representam as frequências numéricas calculadas pelo programa ANSYS. A função $\mathrm{F}_{\text {objt }}$ é função a ser minimizada, na qual é descrita como:

$$
F_{o b j t}=\sum_{i=1}^{n}\left(\frac{f_{n u m}^{i}-f_{e x p}^{i}}{f_{e x p}^{i}}\right)^{2}
$$

- Onde $f_{n u m}^{i}$ é a frequência natural numérica para o modo de vibração i e, $f_{\text {exp }}^{i}$ é a frequência experimental do mesmo modo i;

- As variáveis de estado e a função objetivo são dependentes das variáveis de projeto e aproximadas por meio de 
ajustes de mínimos quadrados. Cada interação da aproximação por subproblema gera um 'loop' completo de análise por MEF. A convergência ocorre quando há um projeto factível e quando uma das seguintes condições é satisfeita:

$$
\begin{gathered}
\left|F_{o b j t}^{(j)}-F_{o b j t}^{(j-1)}\right| \leq \tau\left|F_{o b j t}^{(j)}-F_{o b j t}^{(j-1)}\right| \leq \tau ; \\
\left|F_{o b j t}^{(j)}-F_{o b j t}^{(b)}\right| \leq \tau\left|F_{o b j t}^{(j)}-F_{o b j t}^{(b)}\right| \leq \tau ; \\
\left|x^{(j)}-x^{(j-1)}\right| \leq \rho_{i}(i=1,2 \ldots, n) \\
\left|x^{(j)}-x^{(b)}\right| \leq \rho_{i},(i=1,2 \ldots, n) .
\end{gathered}
$$

Onde $\mathrm{x}^{(\mathrm{j})}, \mathrm{x}^{(\mathrm{j}-1)} \mathrm{e} \mathrm{x}^{(\mathrm{b})}$ representam à interação atual, anterior e melhor, respectivamente, com como as tolerâncias da $\mathrm{F}_{\text {objt }}$ e das variáveis de estado;

Caso nenhuma das equações anteriores seja satisfeita, o programa recorre a outras duas condições para encerrar as interações. A primeira condição é a igualdade entre o número de interações por subproblema com o número máximo de interações, e a segunda corresponde à igualdade entre o número de passos sequenciais impossíveis com o número máximo de passos sequenciais possíveis.

Para os cálculos no ANSYS, considerou-se a placa fina de CARALL como um contínuo, isto é, as cinco lâminas que o constituem são supostas como uma única lâmina. Portanto, os valores atribuídos a placa se resumem as duas dimensões [(300x200) $\mathrm{mm}]$ e uma única massa específica $\left(2,51 \mathrm{~g} / \mathrm{cm}^{3}\right)$. Simplificando assim a modelagem no ANSYS e processo de otimização que ficou dependente somente das frequências naturais obtidas no ensaio de vibração em placa livre. A mesma analogia dimensional foi aplicada para a placa de alumínio.

No caso do CARALL, optou-se por uma malha do tipo "SHELL99" com 196 elementos de 8 nós cada. Cada frequência calculada pelo método dos elementos finitos para essas placas retangulares se relaciona com um modo de vibração diferente. E cada constante elástica está mais suscetível a um determinado modo de vibração puro ou combinado (Figura 3) [11]. Como não se sabia quais os valores para as constantes elásticas, arbitraram-se seis valores diferentes para cada propriedade elástica, executando o programa por cinco vezes, gerando, portanto, cinco grupos que após otimização forneceu uma média para os valores desejados $\left(\mathrm{E}_{1}, \mathrm{E}_{2}, \mathrm{G}_{12}\right.$ e $\left.v_{12}\right)$. Para a placa de alumínio, como se trata de um material isotrópico, somente duas constantes elásticas são o suficiente para descrever o seu comportamento mecânico.
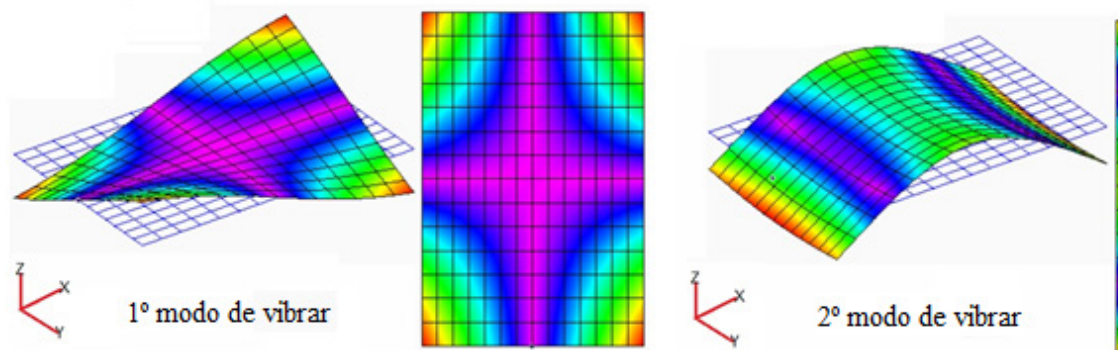

$2^{\circ}$ modo de vibrar
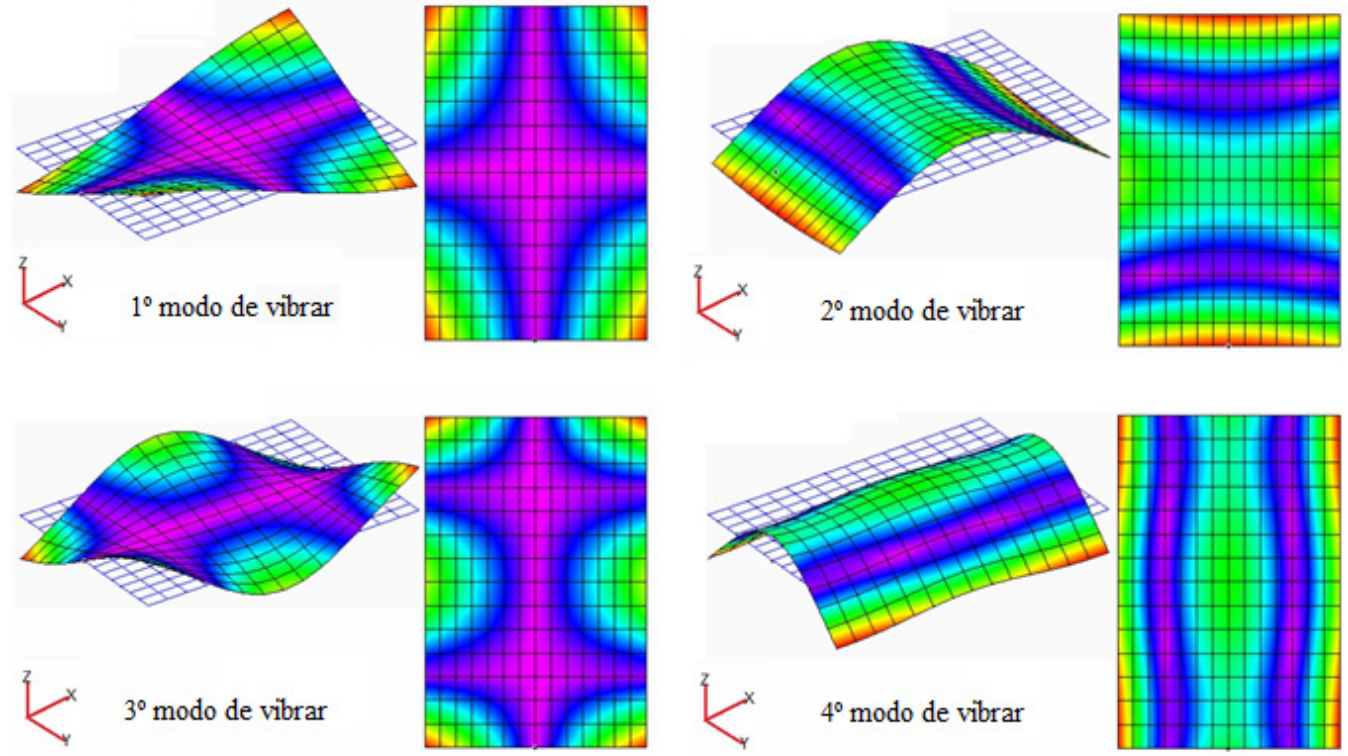

Figura 3: Exemplo de alguns modos de vibrar para uma placa retangular de um material ortotrópico.

Na programação em linguagem APDL do ANSYS foram atribuídos valores para os seus comandos relacionados às tolerâncias e limites usados neste processo interativo. Estes valores são apresentados, como exemplo, na Tabela 1 para o CARALL. 
Tabela 1: Tolerâncias e limites para as otimizações da placa de CARALL

\begin{tabular}{l|c|c}
\hline \multicolumn{1}{c}{ MATERIAL: CARALL } & Comando no APDL & Valor \\
\hline Tolerância para variável de estado $f_{\text {num }}, \mathrm{Hz}$ & tolsv & 0,5 \\
Tolerância para variável de projeto $\mathrm{E}_{1}, \mathrm{MPa}$ & toldvE1 & 50 \\
Tolerância para variável de projeto $\mathrm{E}_{2}, \mathrm{MPa}$ & toldvE2 & 50 \\
Tolerância para variável de projeto $\mathrm{G}_{12}, \mathrm{MPa}$ & toldvG1 & 50 \\
Tolerância para variável de projeto $v_{12}, \mathrm{MPa}$ & toldvv1 & 0,01 \\
Tolerância para função objetivo & tolobj & 0,001 \\
Limite mínimo para a variável de estado $f_{\text {num }}, \mathrm{Hz}$ & tolmin & $0,98\left(f_{\text {num }}\right)$ \\
Limite máximo para a variável de estado $f_{n u m}, \mathrm{~Hz}$ & tolmax & $1,02\left(f_{\text {mum }}\right)$ \\
Limite mínimo para a variável de projeto $\mathrm{E}_{1}, \mathrm{~Hz}$ & vmindvE1 & 60000 \\
Limite máximo para a variável de projeto $\mathrm{E}_{1}, \mathrm{~Hz}$ & vmaxdvE1 & 90000 \\
Limite mínimo para a variável de projeto $\mathrm{E}_{2}, \mathrm{~Hz}$ & vmindvE2 & 60000 \\
Limite máximo para a variável de projeto $\mathrm{E}_{2}, \mathrm{~Hz}$ & vmaxdvE2 & 90000 \\
Limite mínimo para a variável de projeto $\mathrm{G}_{12}, \mathrm{~Hz}$ & vmindvG1 & 10000 \\
Limite máximo para a variável de projeto $\mathrm{G}_{12}, \mathrm{~Hz}$ & vmaxdvG1 & 30000 \\
Limite mínimo para a variável de projeto $v_{12}$ & vmindvv1 & 0,20 \\
Limite máximo para a variável de projeto $v_{12}$ & vmaxdvv1 & 0,40 \\
\hline
\end{tabular}

\section{RESULTADOS}

As frequências experimentais obtidas nos ensaios de vibração livre para os dois tipos de placa de teste (alumínio aeronáutico AA2024-T3 e o compósito híbrido CARALL) são apresentados na Tabela 2. Para verificar a precisão dos resultados, as novas frequências numéricas obtidas com a introdução dos valores médios encontrados para as constantes elásticas foram comparadas com as frequências obtidas no experimento de vibração não amortecida para cada placa teste.

Tabela 2: Frequências experimentais obtidas via vibração não amortecida em comparação com as frequências numéricas calculadas via interação computacional empregando o método de elementos finitos MEF

\begin{tabular}{|c|c|c|c|c|c|c|c|}
\hline & Modos de vibração/ FRF & $1^{\circ}$ & $2^{\circ}$ & $3^{\circ}$ & $4^{\circ}$ & $5^{\circ}$ & $6^{\circ}$ \\
\hline \multirow{3}{*}{ AA2024-T3 } & Frequência experimental (Hz) & 350,86 & 382,39 & 805,77 & 880,17 & 1005,63 & 1179,94 \\
\hline & Frequência numérica $(\mathrm{Hz})$ via $\mathrm{MEF}$ & 349,44 & 377,68 & 806,00 & 881,43 & 1011,80 & 1178,40 \\
\hline & Diferença percentual (\%) & 0,41 & 1,23 & 0,03 & 0,14 & 0,61 & 0,13 \\
\hline \multirow{3}{*}{ CARALL } & Frequência experimental (Hz) & 79,56 & 102,52 & 196,42 & 231,47 & 249,35 & 302,46 \\
\hline & Frequência numérica $(\mathrm{Hz})$ via $\mathrm{MEF}$ & 79,79 & 100,53 & 192,57 & 226,93 & 255,98 & 308,51 \\
\hline & Diferença percentual (\%) & 0,29 & 1,94 & 1,96 & 1,96 & 2,66 & 2,00 \\
\hline
\end{tabular}

A Tabela 3 mostra os valores iniciais arbitrados para os grupos de variáveis de projeto para os dois tipos de materiais. A partir desses grupos foram gerados os resultados apresentados na Tabela 4.

Tabela 3: Valores iniciais arbitrados para os grupos de variáveis de projeto usados na otimização computacional

\begin{tabular}{c|c|c|c|c|c|c}
\hline MATERIAL & $\begin{array}{c}\text { VARIÁVEIS DE } \\
\text { PROJETO }\end{array}$ & GRUPO I & GRUPO II & GRUPO III & GRUPO IV & GRUPO V \\
\hline \multirow{2}{*}{ AA2024-T3 } & $\mathrm{E}_{1}(\mathrm{MPa})$ & 700 & 10.000 & 30.000 & 50.000 & 68.000 \\
& $v_{12}$ & 0,2 & 0,23 & 0,28 & 0,32 & 0,36 \\
\hline \multirow{3}{*}{ CARALL } & $\mathrm{E}_{1}(\mathrm{MPa})$ & 800 & 10.000 & 30.000 & 72.000 & 80.000 \\
& $\mathrm{E}_{2}(\mathrm{MPa})$ & 800 & 9.000 & 28.000 & 70.000 & 78.000 \\
& $\mathrm{G}_{12}(\mathrm{MPa})$ & 500 & 3.000 & 7.000 & 16.000 & 20.000 \\
& $v_{12}$ & 0,2 & 0,23 & 0,28 & 0,36 & 0,42 \\
\hline
\end{tabular}


Tabela 4: Resultado das seis otimizações promovidas para as placas planas de materiais para uso aeronáutico

\begin{tabular}{c|l|c|c|c|c|c|c}
\hline MATERIAL & $\begin{array}{c}\text { VARIÁVEIS DE } \\
\text { PROJETO }\end{array}$ & GRUPO I & GRUPO II & GRUPO III & GRUPO IV & GRUPO V & MÉDIA \\
\hline \multirow{3}{*}{ AA2024-T3 } & $\mathrm{E}_{1}(\mathrm{MPa})$ & 71.582 & 71.276 & 70.852 & 71.492 & 71.374 & $\mathbf{7 1 . 3 1 5}$ \\
& $v_{12}$ & 0,2745 & 0,3390 & 0,3376 & 0,3199 & 0,2766 & $\mathbf{0 , 3 1 0}$ \\
& $F_{\text {objit }}\left(10^{-4}\right)$ & 5,88 & 5,34 & 6,15 & 2,26 & 5,26 & - \\
\hline \multirow{5}{*}{ CARALL } & $\mathrm{E}_{1}(\mathrm{MPa})$ & 70.258 & 69.976 & 70.234 & 70.207 & 70.203 & $\mathbf{7 0 . 1 7 6}$ \\
& $\mathrm{E}_{2}(\mathrm{MPa})$ & 63.146 & 63.268 & 63.490 & 63.275 & 63.475 & $\mathbf{6 3 . 3 3 1}$ \\
& $\mathrm{G}_{12}(\mathrm{MPa})$ & 19.050 & 19.077 & 19.158 & 18.908 & 19.035 & $\mathbf{1 9 . 0 4 6}$ \\
& $v_{12}$ & 0,396 & 0,385 & 0,386 & 0,375 & 0,377 & $\mathbf{0 , 3 8 4}$ \\
\cline { 2 - 8 } & $F_{\text {obji }}\left(10^{-4}\right)$ & 2,28 & 2,27 & 2,26 & 2,28 & 2,27 & - \\
\hline
\end{tabular}

\section{DISCUSSÃO}

Verificou-se que as frequências experimentais da placa de alumínio apresentaram menor diferença porcentual (de $0,03 \%$ a $0,61 \%$ ) em comparação com as suas frequências obtidas pelo MEF (Tabela 2), provavelmente devido seu caráter isotrópico.

As seis frequências experimentais que caracterizam os seis primeiros modos de vibração do compósito CARALL foram inseridas no processo interativo resultando, em média, nas seguintes constantes elásticas: $\mathrm{E}_{1}=70,2 \mathrm{GPa}$; $\mathrm{E}_{2}=63,3$ $\mathrm{GPa} ; \mathrm{G}_{12}=19 \mathrm{GPa}$ e $v_{12}=0,38$. A diferença entre os valores de $E_{1}$ e $E_{2}$, apesar de ter se usado tecido de fibra de carbono com configuração plain weave, pode ter sido uma consequência da geometria adotada para a placa do experimento (retangular ao invés de quadrada). Isso foi verificado por Tan e Colaboradores [19] em um estudo numérico via método dos elementos finitos tridimensional. Em relação ao módulo elástico $\mathrm{E}_{1}$ o valor é próximo do calculado pela regra da mistura (70 GPa) obtida pela equação adaptada ao compósito hibrido do tipo FML, ou seja, $E_{F M L}=E_{\text {Aluminio }} \cdot V_{\text {Aluminio }}+E_{\text {compósito }} \cdot\left(1-V_{\text {Aluminio }}\right)$. O resultado obtido por ensaio de vibração livre-livre para o CARALL se diferencia dos valores observados em ensaios de tração quase-estáticos $\left(\mathrm{E}_{1} \approx 59 \mathrm{GPa}\right.$, por exemplo).

Considera-se ainda, uma limitação desta técnica de vibração não amortecida quanto o uso do conjunto do stinger com o transdutor de força. Ou seja, é possível haver uma interferência nos modos de vibração devido a massa do transdutor de força ( 28 gramas) que fica colado atrás da placa teste. E essa interação entre a massa do transdutor de força e a massa da placa pode aumentar quanto mais leve, ou menor massa tiver a placa teste. O dispositivo stinger também merece atenção. Neste caso, foi usado um stinger com diâmetro de $2,5 \mathrm{~mm}$ e, para uma placa mais leve, sugere-se empregar um stinger com menor diâmetro para verificar se o mesmo poderia interferir nos modos de vibração.

\section{CONCLUSÕES}

As constantes elásticas obtidas pelo processo de otimização com base nas frequências experimentais e numéricas para o material CARALL foram próximos aos valores teóricos encontrados na literatura. Teoricamente, esta regra serve bem ao propósito de referência para validação do resultado obtido, apesar de se tratar de um método linear simplificado, que deixa de considerar alguns fatores importantes, como por exemplo, a adesão interfacial entre as lâminas do compósito híbrido. Porém, em geral, os resultados se mostraram satisfatórios principalmente os valores para os módulos de elasticidade transversal e longitudinal. As frequências numéricas correspondentes a segunda e quarta forma modal (modos de flexão puro) que exercem significativa influência nas constantes $\mathrm{E}_{1}$ e $\mathrm{E}_{2}$, estão com uma boa aproximação das frequências obtidas no ensaio. Evidentemente, não se pode referenciar as frequências experimentais como "parâmetros ideais" uma vez que este ensaio de placas pode estar sujeito a alguns fatores que podem induzir erros na medição. Um exemplo disso é o acréscimo de massa pontual pelo transdutor de força, uma vez que o ideal seria a placa não ter nenhum dispositivo físico em contato.

\section{AGRADECIMENTOS}

A Fundação de Amparo à Pesquisa e ao Desenvolvimento Científico e Tecnológico do Maranhão FAPEMA e ao IAE/DCTA pelo suporte técnico dos ensaios.

\section{BIBLIOGRAFIA}

[1] PARDINI, L.C., NETO, F.L., Compósitos estruturais: Ciência e Tecnologia, 1 ed, São Paulo, Edgard Blüncher, 2006.

[2] VERMEEREN, C.A.J.R., "An historic overview of the development of fibre metal laminates", Applied Composite 
Materials, v.10, n.4-5, pp.189-205, Jul. 2003.

[3] GONZALEZ-CANCHE, N.G., FLORES-JOHNSON, E.A., CARRILLO, J.G. "Mechanical characterization of fiber metal laminate based on aramid fiber reinforced polypropylene”, Composite Structures, v.172, pp.259-266, Jul. 2017.

[4] ZHANG, J. et al. "Characterizing the off-axis dependence of failure mechanism in notched fiber metal laminates", Composite Structures, v.185, pp.148-160, Fev. 2018.

[5] BOSBACH, B, et al. "Maximizing bearing fatigue lifetime and CAI capability of fibre metal laminates by nanoscale sculptured Al plies”. Composites Part A: Applied Science and Manufacturing, v.117, pp.144-155, Fev. 2019.

[6] YAO, L. et al. "Investigation on impact behavior of FMLs under multiple impacts with the same total energy: Experimental characterization and numerical simulation”, Composite Structures, n.226, pp.1-23, Jul. 2019.

[7] KAZEMI, M.E., SHANMUGAM, L., YANG, L., YANG, J. "A review on the hybrid titanium composite laminates (HTCLs) with focuses on surface treatments, fabrications, and mechanical properties", Composites Part A: Applied Science and Manufacturing, v.128, pp.1-16, Jan.2020.

[8] RÍOS, J. C. et al. "Determination of fracture toughness J on fiber-metal laminate type CARALL with sheets of aluminium 6061”, Procedia Materials Science, v.9, pp.530 - 537, 2015.

[9] BOTELHO, E.C., SILVA, R.A., PARDINI, L.C., REZENDE, M.C. “A Review on the Development and Properties of Continuous Fiber/epoxy/aluminum Hybrid Composites for Aircraft Structures”, Materials Research, v.9, n.3, pp.247-256, Set.2006

[10] SASSO, M. et al. "Investigation of the mechanical behavior of CARALL FML at high strain rate", Composite Structures, v.222, pp.1-17, Ago.2019.

[11] BRITO JÚNIOR, C.A.R., Caracterização Dinâmico-Mecânica de Compósitos Metal/Fibra e Compósitos de Fibras de Carbono/Resina Epóxi para Uso Aeronáutico. Tese de M.Sc., ITA, São José dos Campos, SP, 2007.

[12] DAVIS, J. R., ASM Specialty Handbook: Aluminum e and Aluminum Alloys, Ohio, ASM International, 1993.

[13] HEXCEL Composites, Prepreg Technology, Duxford, UK, 1997. Publication No FGU 017.

[14] GIBSON, R.F., Principles of composite materials mechanics, New York, McGraw Hill, 1994.

[15] FREDERIKSEN, P.S., "Single-layer plate theories applied to flexural vibration of completely free thick laminates", Journal of Sound and Vibration, v.186, n.5, pp.743-759, Out. 1995.

[16] HWANG, S.-F., CHANG, C.-S., "Determination of elastic constants of materials by vibration testing", Composite Structures, v.49, n.2, pp.183-190, Jun. 2000.

[17] BATISTA, F.B., Identificação de parâmetros elásticos em placas finas de materiais compósitos, F191, 2006. Tese de M.Sc., FEM/UNICAMP, Campinas, SP, Brasil, 2006.

[18] PAIVA, J.M.F., MAYER, S., REZENDE, M.C. "Comparison of Tensile Strength of Different Carbon Fabric Reinforced Epoxy Composites”, Materials Research, v.9, n.1, pp.83-89, Jan./Mar.2006.

[19] TAN, P., TONG, L., STEVEN, G. P. “A flexible 3D FEA modelling approach for predicting the mechanical properties of plain weave unit cell”, In: Proceedings of Eleventh International Conference on Composite Materials, v.5, pp.67-76, 1997.

ORCID

Carlos Alberto Rios Brito Júnior Luis Claudio Pardini Antônio Carlos Ancelotti Júnior https://orcid.org/0000-0003-0640-8165

https://orcid.org/0000-0001-6982-1577

https://orcid.org/0000-0003-2031-419X 
